is only assured at low temperatures. Vibrational spectroscopic examination of gaseous reaction products quenched to liquid air or lower temperatures probably offers the best opportunity to observe the $\mathrm{HeBeO}$ species. It could be that $\mathrm{HeBeO}$ would be an effective source of ' $\Sigma^{+} \mathrm{BeO}$, if the lifetime of the triatomic, as seems likely, is greater than that of the diatomic. (Although strongly bound as a diatomic, $\mathrm{BeO}$ - like $\mathrm{LiF}$ and $\mathrm{BN}$ - rapidly polymerizes and cannot be isolated.) It could be, however, that the most important aspect of this discovery is the superacceptor $\left(\sigma\right.$-acid) property of ${ }^{\prime} \Sigma^{+} \mathrm{BeO}$. This molecule will surely form Lewis acid-base adducts with many molecules other than helium. Even the poor bases (poor electron donors) $\mathrm{CO}$ and $\mathrm{N}_{2}$ should form the stable linear tetra-atomics $\mathrm{OCBeO}$ and NNBeO.

Neil Bartlett is in the Department of Chemistry, University of California, Berkeley, California 94720, USA.

\title{
Supernovae
}

\section{Fewer and further between}

\section{Virginia Trimble}

THE rate at which supernova explosions occur enters into our models of galactic evolution, interstellar gas dynamics and other phenomena. And the rates we have been using are too big by a factor of about three. So conclude ${ }^{1}$ van den Bergh, McClure and Evans on the basis of systematic searches for supernovae in bright southern galaxies by Australian amateur Robert Evans. Five years' surveillance led to 15 discoveries, including six type II events (with hydrogen lines in their spectra) and nine type I (lacking hydrogen). But, according to the authors' interpretation of conventional rates ${ }^{2}$ and of the search parameters, 54 supernovae should have turned up six type IIs and 48 type Is, a significant discrepancy.

What rates should we be using? The answer from van den Bergh et al. ' applies to galaxies in the Shapley-Ames Catalog ${ }^{3}$, which contains a mix of types but is dominated by spirals rather like our Milky Way. For these, the total rate is $1.8 h^{2}$ per century per $10^{10}$ solar luminosities in a blue waveband $\left(10^{10} L_{\odot}^{\mathrm{B}}\right)$. The normalization by luminosity is clearly needed - bright galaxies will have more explosion-prone stars than will faint ones. The parameter $h=H_{0} /\left(100 \mathrm{~km} \mathrm{~s}^{-1} \mathrm{Mpc}^{-1}\right)$, where $H_{0}$ is your favourite value of Hubble's constant, expresses our uncertainty about distances to external galaxies and hence about their actual luminosities. The authors divide the $1.8 h^{2}$ supernovae among $1.1 h^{2}$ type II, $0.3 h^{2}$ type Ia (no hydrogen; found in old stellar populations), and $0.4 h^{2}$ type Ib (no hydrogen, but found in young populations) per century per $10^{10} L^{\mathrm{B}}$.

For our own Galaxy, van den Bergh et al. adopt ${ }^{1}$ a luminosity of $2 \times 10^{10} L^{\mathrm{B}}$ and so predict rates of $2.2,0.6$ and $0.8 h^{2}$ per century for the three types, or one supernova every 28 yr (because they belong to the $h \simeq 1.0$ school of thought). Other constraints on the galactic rate are, at least, not inconsistent. Pulsars, generally supposed to come from type II supernovae, are born every 60 yr (ref. 4). Supernova remnants, which come from supernovae surrounded by relatively dense gas, form every $60-80$ yr (refs 5,6$)$. Both these intervals probably have factor-of-two uncertainties. Finally, the seven supernovae seen in our sector of the Milky Way since 1006 AD imply an interval of about $30 \mathrm{yr}$ between events over the whole Galaxy. If these numbers sound familiar, it is because van den Bergh et al. find almost no discrepancy with earlier results for the particular case of the Milky Way. Tammann expected $^{2} 4.2 h^{2}$ supernovae per century per $10^{10} L^{B}$ in a galaxy of our type. But he adopted $h=0.5$ and a galactic luminosity of $4 \times 10^{10} L^{\mathrm{B}}$. Thus, after also considering the historical events, he concluded that a galactic supernova occurs every 20-25 yr.

Predictably, the new lower rate is neither entirely unexpected nor entirely straightforward to interpret. In the past few years, several groups have concluded that supernovae as numerous as previously advertised would put more heat, kinetic energy and other disturbances into interstellar material then observations permit in E (elliptical) and S0 (lenticular) galaxies and other spirals ${ }^{8.9}$ as well as in the Milky Way ${ }^{10}$. Downward revisions by factors of two to three were required in all cases. The new rates seem to be just right.

They also fit well with a calibration based on deriving the birth rate of massive stars from the Lyman continuum photons which they produce and then asking how far down in mass one has to go among these stars to produce a given supernova rate $^{11}$. For the previous, high, rate, this was about five solar masses, down into the range that we think, on other grounds, actually makes white dwarfs rather than supernovae. Curiously, the new rate is not very different from that proposed by Zwicky $^{12}$ on the basis of his first three supernovae, found in 1,750 galaxy-years of searching. Because his search focused on members of nearby small groups, his average galaxy had $L=10^{9} h^{-2} L_{\odot}^{\mathrm{B}}$ and his discovery rate corresponds to about $1.7 h^{2}$ supernovae per century per $10^{10} L^{\mathrm{B}} \odot$.
There remains one conceptual difficulty in interpreting the difference between the old and new rates. Type I events (at least type Ia events) are brighter than type IIs by about one magnitude or a factor of 2.5 . Clearly this introduces some sort of differential effect into most searches, the fainter type II supernovae being discriminated against. Tammann made no attempt ${ }^{2}$ to allow for this, assuming that all supernovae of both types in his fiducial galaxies and observation interval had been found. Thus his ratio of type I to type II supernovae in spiral galaxies is 1.2 , the observed ratio in about 80 events actually recorded and typed. I would, therefore, have expected Evans to find a similar ratio, as indeed he did $-9 / 6=1.5$.

But the ratio predicted by van den Bergh et al. is $44 / 6$ or 7.3 . What has happened is that they started with Tammann's rates, acted as if these had been corrected for a differential observational selection effect, and corrected back. The authors assumed Evans' search was complete to a particular level of apparent brightness (probably correct), calculated how many events should have risen above that level in the search period, and so concluded that the brighter type I supernovae should greatly outnumber the fainter type IIs on the discovery list. This was clearly to some extent the wrong thing to do. I do not know how precisely to describe the right algorithm, but it ought to predict roughly equal discovery rates for type I and type II supernovae in spiral galaxies.

Luckily this problem does not affect the reliability of the supernova rates finally adopted by van den Bergh et al.. These were derived by starting with the actual discovery list and allowing for differential selection effects. The result is that an observed type I to type II ratio of 1.5 is transformed into an occurrence ratio of 0.6 . The main conclusion seems also to stand firm: Evans' predecessors were more thorough supernova searchers than we have given them credit for, and so the rates we have been using, derived from their work, were too large by factors of two to three.

1. van den Bergh. S., McClure, R.D. \& Evans, R. Astrophys. J. 323, 44 (1987)

. Tammann. G.A. in Supernovae: A Survey of Currem Research (eds Rees, M.J. \& Stoneham. R.J.) 371 (Reidel. Dordrecht, 1982)

3. Sandage, A.R. \& Tammann, G.A. A Revised Shapley. Ames Catalog (Carnegie Instn, Washington, DC, 1981).

4. Lyne, A.G., et al. Mon. Not. R. astr. Soc. 213,613 (1985)

5. Caswell, J.L. \& Lerche, I. Mon. Not. R. astr. Soc. 187.201 (1979).

6. Green. D.A. Mon. Not. R. astr. Soc. 216, 691 (1985)

7. Canizares. C. et al. Astrophys. J. 312. 503 (1987).

8. Brinks, E. \& Bajaja, E.M. Astr. Astrophys. 169, 14 (1986).

9. Cox, D.P. \& McCammon, D. Astrophys. J. 304. 657 (1986).

10. Heiles, C. Astrophys, J. 315, 555 (1987).

11. Berkhuijsen, E.M. Astr. Astrophys. 140, 431 (1984).

12. Zwicky, F. Astrophys. J. 88, 529 (1938)

Virginia Trimble is Professor of Physics at the University of California, Irvine, California 92717, and is Visiting Professor of Astronomy at the University of Maryland, College Park, Maryland 20742, USA. 